\title{
Distribution of $\{110\}\langle 001\rangle$ Oriented Grains in the Primary Recrystallized 3\% Si-Fe Alloy*
}

\author{
By Jirou Harase** and Ryo Shimizu**
}

\begin{abstract}
The distribution of $\{110\}\langle 001\rangle$ oriented grains in the primary recrystallized $3 \% \mathrm{Si}-\mathrm{Fe}$ alloy processed by the one-stage cold rolling method were investigated by SEM-ECC-ECP technique and the X-ray diffraction method. The main findings were in the following:

(1) $\{110\}\langle 001\rangle$ oriented grains present below $30 \mu \mathrm{m}$ from the surface of the primary recrystallized specimen were not among the largest grains nor were present as an aggregate. The frequency of the $\{110\}$ grains rotated about $0.35 \mathrm{rad}$ around the ND axis had the highest frequency among the $\{110\}$ oriented grains and the frequency of an ideal Goss orientation is less than $1 / 3$ of these orientations. Orientations of the extraordinarily large grains were similar to the major textural component of the primary recrystallized specimen.

(2) Among the $\{110\}$ oriented grains, the Goss oriented grain had the highest frequency of coincidence oriented grains in the grains directly surrounding to these $\{110\}$ oriented grains and had a very low frequency of the $\Sigma 1$ boundary in relation to its orientation in the surface of the primary recrystallized matrix, while the large grains above mentioned had a rather higher frequency of the $\Sigma 1$ boundary in relation to its orientation.

(3) The mechanism of the evolution of the Goss texture by secondary recrystallization can be explained, even if the Goss nucleus is not the largest at the completion of the primary recrystallization, by considering the special distribution of coincidence boundaries and their specific grain boundary migration characteristics associated with the intensity of the inhibitor.
\end{abstract}

(Received January 28, 1988)

Keywords: secondary recrystallization, grain boundary, precipitation, inhibitor, aluminum nitride, coincidence boundary, grain boundary migration

\section{Introduction}

Grain oriented silicon steels are characterized by the presence of a sharp $\{110\}<100\rangle$ texture, i.e. the Goss texture ${ }^{(1)}$. The deviation angle of $\langle 100\rangle$ axis of grains from the rolling direction is smaller than $10^{\circ}$ for commercially produced silicon steels. Although many investigations have been made in the decades to clarify the mechanism of the evolution of the Goss texture ${ }^{(1)-(10)}$, it is not fully understood why such a sharp Goss texture is evolved by secondary recrystallization. One stream of the investigation has been focused on the Goss nucleus in the primary texture $\mathrm{e}^{(2)(6)-(8)(11)}$.

* A part of this paper was originally published in Japanese in J. Japan Institute of Metals, 52 (1988), 259.

** R \& D Laboratories III, Nippon Steel Corporation, Edamitu, Yawata-Higashi-ku, Kitakyusyu 805, Japan.
Another stream of the investigation has been associated with inhibitors for secondary recrystallization $^{(3)(5)(10)}$. The present authors investigated the distribution of Goss nucleus and the orientation of the grains having large grain size in the surface layer of the primary recrystallized specimen by SEM-ECC-ECP technique and discussed the formation mechanism of Goss texture by secondary recrystallization considering the intensity of the inhibitor and the distribution of the coincidence boundaries in the primary recrystallized specimen.

\section{Experimental Procedure}

The starting material was $2.3 \mathrm{~mm}$ thick hot rolled 3\% silicon steel sheet with the following chemical compositions: C:0.04, Si:2.90, $\mathrm{Mn}: 0.09, \mathrm{~S}: 0.027, \mathrm{Al}: 0.026, \mathrm{~N}: 0.007$ mass $\%$. The primary recrystallized specimen was prepared following the one-stage cold rolling 
method developed by Taguchi et $a l^{(12)}$ as follows:

Hot Band $(2.3 \mathrm{~mm}) \rightarrow$ Annealing $(1393 \mathrm{~K}-$ $150 \mathrm{~s}) \rightarrow$ W.Q (373 K boiling water) $\rightarrow$ Cold Rolling $(0.3 \mathrm{~mm}) \rightarrow$ Annealing $(1039 \mathrm{~K}-150 \mathrm{~s}$, dew point-333 K).

\section{Investigation by SEM-ECC-ECP technique}

The surface layer of the thickness of about $30 \mu \mathrm{m} /$ one side was taken by chemical polishing (1/10 of the total thickness) and this newly made surface was utilized for the SEMECC-ECP investigation (specimen A). The grain size distribution of this surface was investigated by an image analyzer (Tospix II). The total number of grains utilized for the gain size distribution measurement were 789 grains. Then a square of $10 \mathrm{~mm} \times 10 \mathrm{~mm}$ was scribed on this surface so that the one side of the square lay parallel to the rolling direction. Then grains with $\{110\}\langle u v w\rangle$ orientation were scanned by the ECP mode in this area at each $100 \mu \mathrm{m}$ interval along the TD direction.

As the average grain size of the specimen is about $8 \mu \mathrm{m}$ and the pattern is generated from about $5-10 \mu \mathrm{m}$ in diameter, $5-10 \%$ of the 10 $\mathrm{mm} \times 10 \mathrm{~mm}$ square is scanned by ECP by scanning at each $100 \mu \mathrm{m}$ interval. In the selection of patterns, all the grains of $\{110\}\langle u v w\rangle$ orientations in which $\{110\}$ planes less than 0.17 rad deviated from either the TD or RD axis are chosen and for the rotation angle around the ND axis every angles were selected. When the $\{110\}\langle u v w\rangle$ grain was detected by $\mathrm{ECP}$, its grain size was also investigated. 45 grains were arbitrarily chosen from the $\{110\}\langle$ uvw $\rangle$ oriented grains detected by ECP and the orientations of all the grains surrounding these grains were investigated. The coincidence orientation relationship between the surrounding grains and these $\{110\}\langle$ uvw $\rangle$ oriented grains were also investigated. Brandon's condition ${ }^{(13)}$ is used for the criterion of the coincidence orientation relationship. Then grains larger than $20 \mu \mathrm{m}$ in diameter were scanned by the ECC mode under magnifications of $\times 500$ in the same manner as scanned by ECP mode. In this manner all the area of $10 \mathrm{~mm} \times 10 \mathrm{~mm}$ can be scanned. The orienta- tions of these grains were analyzed by ECP. Among these grains, 6 extraordinarily large grains were chosen and the orientation of the surrounding grains and their orientation relationship with these grains were investigated. The scanning electron microscope used was JSM-840. ECP was taken in the following condition; accelerating voltage: $35 \mathrm{kV}$, probe current: $9 \times 10^{-9} \mathrm{~A}$, working distance: $8 \mathrm{~mm}$, rocking angle: $\pm 0.14 \mathrm{rad}$.

\section{Investigation by $X$-ray measurements}

The specimen with $80 \mu \mathrm{m}$ thick including surface was taken for the texture analysis by $\mathrm{X}$ rays (specimen $\mathrm{B}$ ). Transmission and reflection (100) pole figures were made from this specimen and the complete (100) pole figure was made from them. This pole figure was inverted by the vector method ${ }^{(14)}$ and the intensity distribution of $\{110\}\langle 001\rangle$ grains rotated around the ND axis was obtained by the vector method. In order to compare the orientation measured by ECP with that measured by Xrays, the inverse pole figures seen from the ND and the RD direction were made by the vector method. The details of the method of making the RD inverse pole figure are shown elsewhere $^{(15)}$.

\section{Results}

\section{Texture measurements by X-ray}

Figure 1(a) and (b) show the structure and the grain size distribution of specimen $A$. The structure is rewritten for processing by the image analyzer (Tospix II). The grain size distribution was obtained from these structure map consisting of 789 grains by the image analyzer. The average grain size is $7.5 \mu \mathrm{m}$ and that no grains 5 times larger $(=37.5 \mu \mathrm{m})$ than the average grain size is present in this distribution figure.

Figure 2(a) and (b) show (100) pole figure and the inverse pole figure calculated by the vector method of the specimen $B$. It can be seen that the $(100)$ pole figure is a typical pole figure of a primary recrystallized $3 \% \mathrm{Si}-\mathrm{Fe}$ processed by the one-stage cold rolling method. The major textural components are 


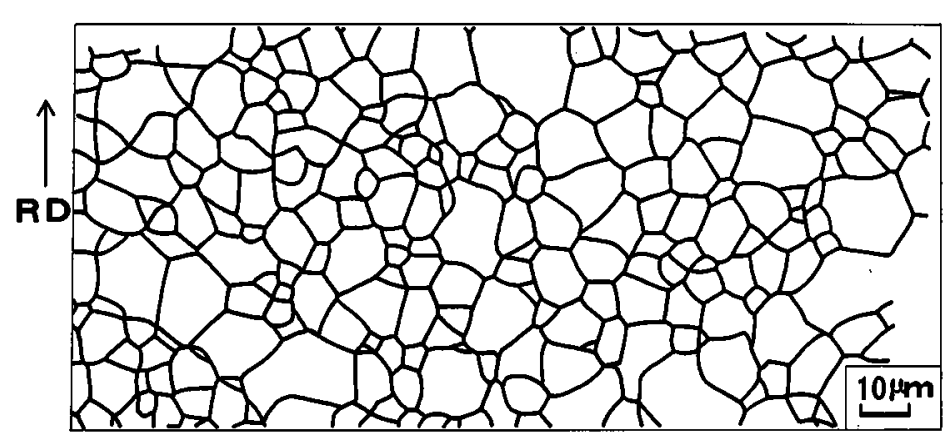

(a) Structure

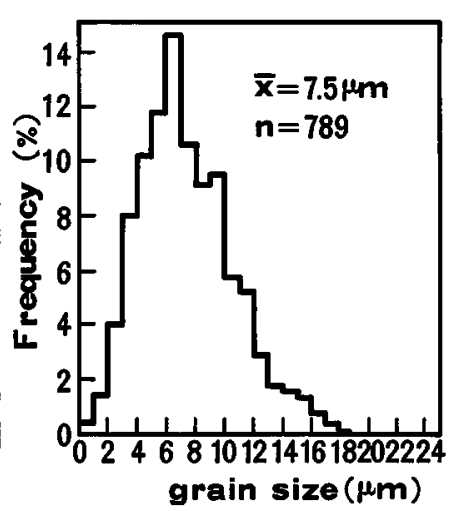

(b) grain size distribution

Fig. 1. (a) The structure and (b) the grain size distribution $30 \mu \mathrm{m}$ below the surface of a primary recrystallized specimen (specimen $\mathrm{A}$ ). The grain size distribution was analyzed by an image analyzer (Tospix II): Numbers of grains utilized are 789.

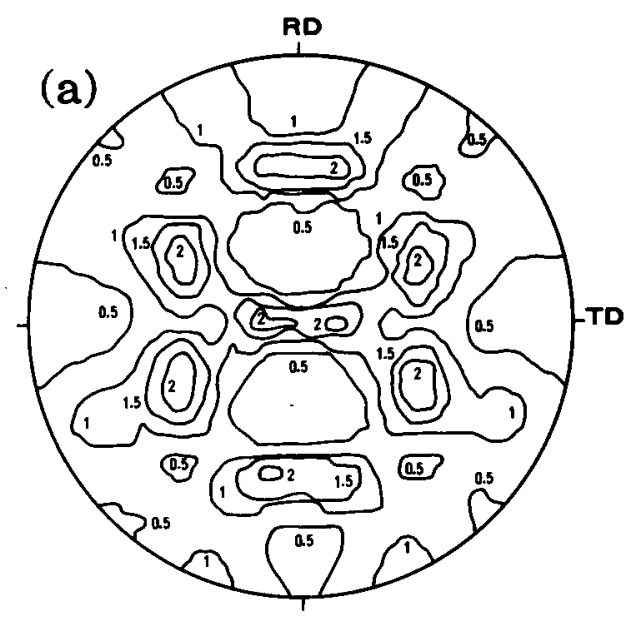

Fig. 2. (a) Complete (100) pole figure and (b) inverse pole figure analyzed by vector method of the surface layer of the primary recrystallized specimen (specimen B).

$\{111\}\langle 112\rangle$ and that the $\{110\}\langle u v w\rangle$ is a minor component.

\section{The distribution of $\{110\}$ grains in the specimen A}

Figure 3 shows the positions of $\{110\}\langle u v w\rangle$ oriented grains detected by ECP below $30 \mu \mathrm{m}$ from the surface of the primary recrystallized

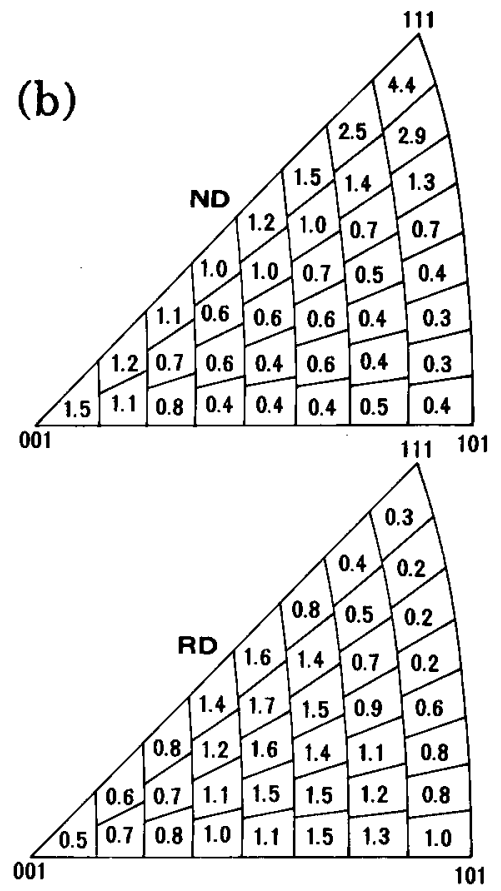

specimen (specimen A). It can be seen that they are rather uniformly distributed. The total numbers of grains detected were 215 . The maximum rotation angle of $\{110\}$ grains from the TD and RD axis was 0.13 and 0.19 rad respectively. Among the $\{110\}\langle u v w\rangle$ oriented grains detected, grains in contact with each other were only three cases and all other $\{110\}\langle$ uvw $\rangle$ 


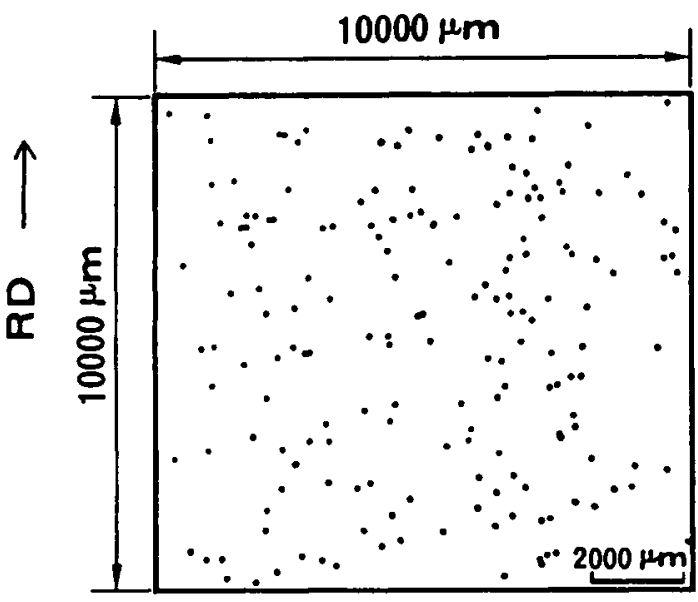

Fig. 3. Positions showing $\{110\}\langle u v w\rangle$ grains detected by ECP present $30 \mu \mathrm{m}$ below the surface of the primary recrystallized specimen (specimen $\mathbf{A}$ ).

oriented grains were present not in contact with each other.

Figure 4 shows grain size and the frequency of $\{110\}$ grains around the ND axis classified by the rotation angle of each $0.087 \mathrm{rad}$ obtained by ECP together with the intensity distribution obtained by the vector method. As the $\{110\}$ planes include the planes rotated around the TD or RD axis up to $0.11 \mathrm{rad}$ in the case of the vector method, the grains of $\{110\}$ planes measured by ECP utilized for this analysis are selected to include the same planes as in the vector method. Frequency distribution obtained by the vector method and ECP is normalized so as to compare them. It can be seen from this figure that the shape of the intensity distribution by the vector method and by ECP is very similar suggesting that the grain size and the distribution of $\{110\}$ oriented grains detected by ECP represents the overall characteristics of the distribution of the $\{110\}$ oriented grains at the $30 \mu \mathrm{m}$ below the surface layer of the primary recrystallized specimen. This result also suggests that the size of the grains having an ideal Goss orientation or nearly ideal Goss orientation (they are orientations of the secondary recrystallized specimen) is not particularly large, although they are slightly larger than the average grain size and they do not exist as colonies. Among the $\{110\}$ oriented grains, the grain rotated about 0.35

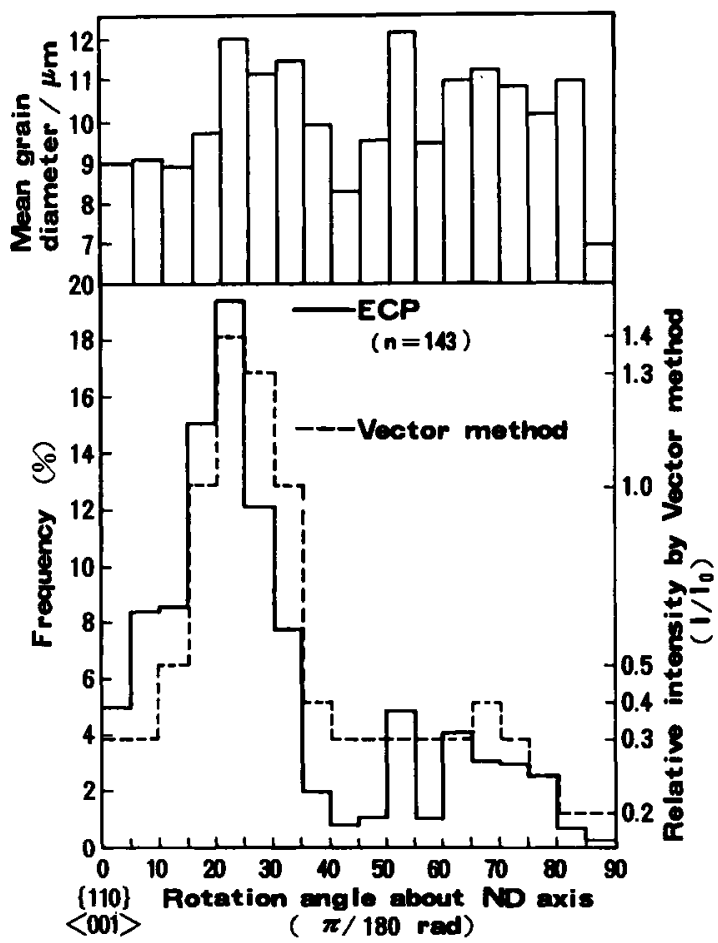

Fig. 4. Grain size distribution of $\{110\}$ grains present 30 $\mu \mathrm{m}$ below the surface of primary recrystallized specimen and the comparison of their frequency distribution determined by ECP and the vector method. Frequency determined by ECP and the vector method are normalized to compare them. Numbers of grains used for ECP analysis are 143 .

rad around the ND axis has the highest intensity and that the intensity of an ideal Goss orientation is less than $1 / 3$ of the maximum intensity.

\section{Orientation and rotation relationship of the grains directly surrounding $\{110\}$ oriented grains}

45 grains were arbitrarily chosen from the $\{110\}$ oriented grains detected by ECP. The orientation and rotation relationship of the grains directly surrounding these grains were investigated by ECP. The number of these grains were 390. Figure 5 shows orientation of 9 grains of nearly ideal Goss orientation and the surrounding grains. Although the number of the grains investigated is small, it can be seen from this figure that the orientations of the surrounding grains are similar to those surround- 


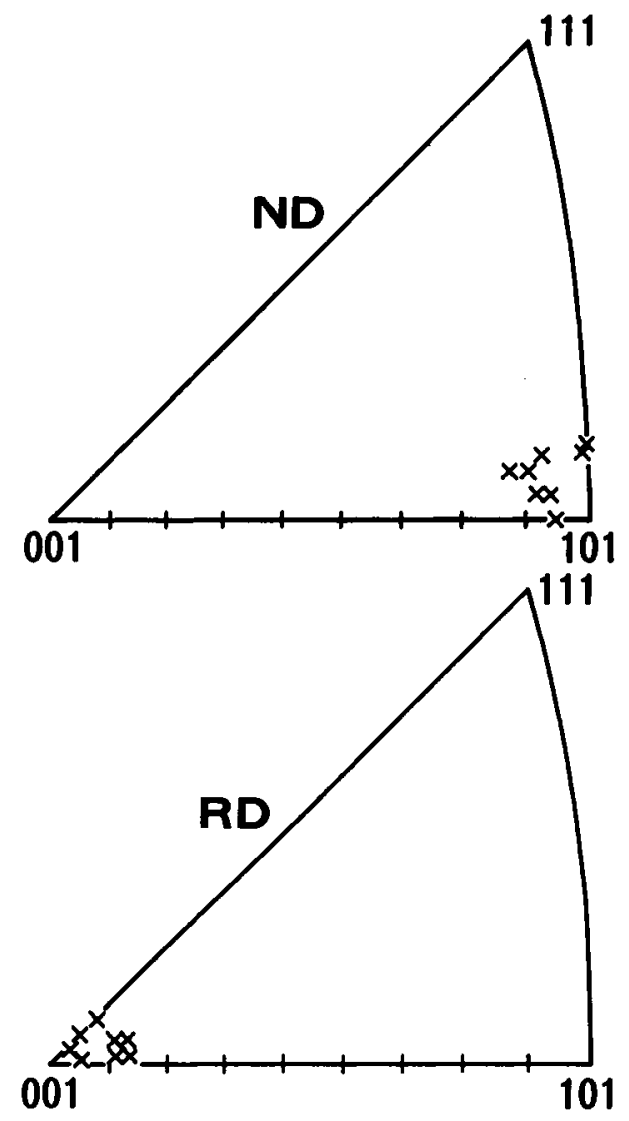

(a) $\{110\}<110>$ grains

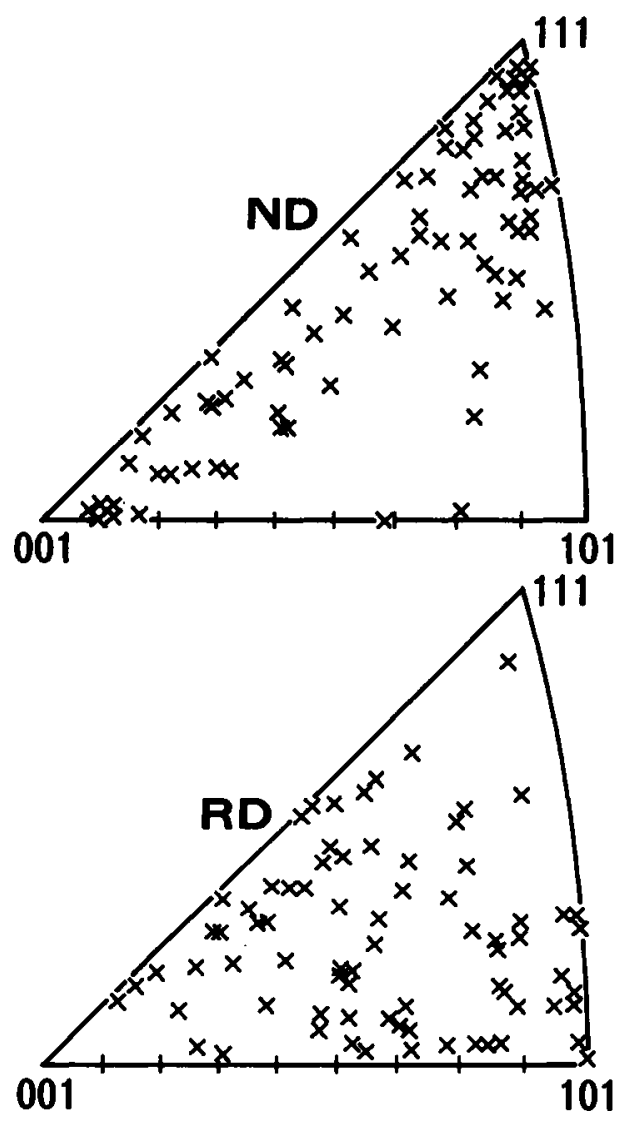

(b) sur rounding grains

Fig. 5. An example showing (a) orientations of $9\{110\}\langle 001\rangle$ grains and (b) grains directly contacted to those $\{110\}\langle 001\rangle$ grains present $30 \mu \mathrm{m}$ below the surface of the primary recrystallized specimen (specimen $\mathrm{A})$.

ing large grains (Fig. 9). Figure 6 shows frequency of coincidence boundaries between the $\{110\}$ oriented grain and its surrounding grains. The $\{110\}$ oriented grains investigated are 45 grains as mentioned above. It can be seen from this figure that the frequency of $\Sigma 5$, $\Sigma 9, \Sigma 19$ and $\Sigma 45$ are relatively high (except $\Sigma 1)$. Figure 7 shows the frequency of coincidence boundaries between the nearly ideal Goss oriented gains (deviation angle of the $\langle 100\rangle$ axis is less than $0.087 \mathrm{rad}$ ) and their surrounding grains, and the $\{110\}$ oriented grains largely deviated from the ideal Goss orientation (deviation angle of the $\langle 100\rangle$ axis is larger than $0.28 \mathrm{rad}$ ) and their surrounding grains. Although the number of $\{110\}$ grains utilized for this investigation are small (43 grains), it shows that nearly ideal Goss oriented grains have the highest frequency of coincidence boundaries.

\section{Orientation and rotation relationship of the grains directly surrounding a large grain}

Figure 8 shows orientation and grain size distribution of all the grains larger than $20 \mu \mathrm{m}$ in diameter present in the same area scanned by ECP in specimen A. As the maximum grain size in the grain size distribution shown in the Fig. 1(b) is $20 \mu \mathrm{m}$, the large grains detected by this method is considered to be extraordinarily large grains in this specimen. Among the 


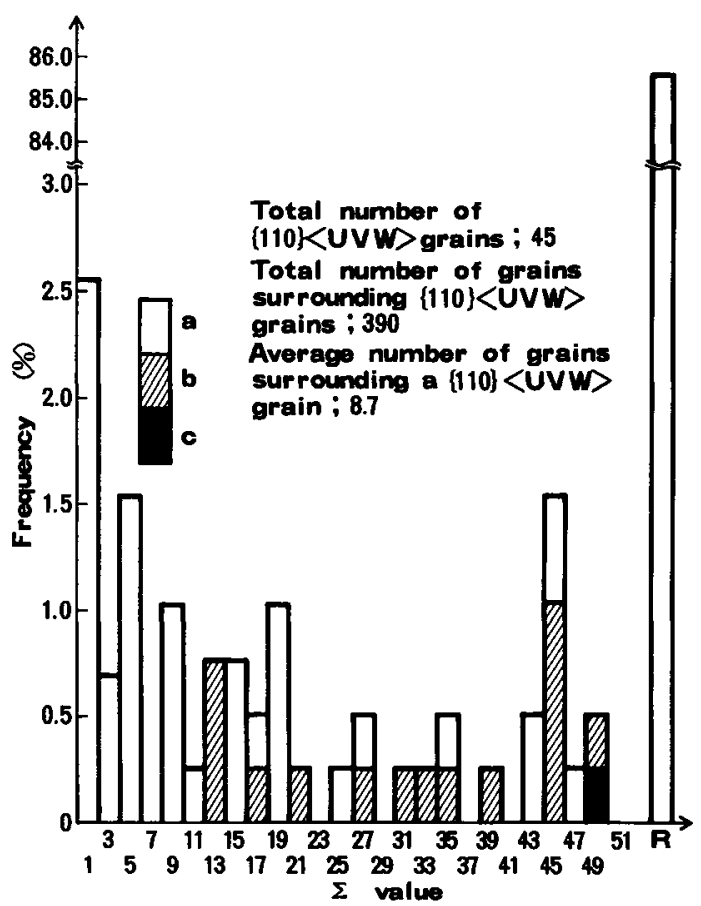

Fig. 6. Frequency of coincidence boundaries surrounding $\{110\}\langle\mathrm{uvw}\rangle$ grains present at the $30 \mu \mathrm{m}$ below the surface of the primary recrystallized specimen (specimen A, R; random boundaries). grains detected, no grains of nearly Goss orientation (deviation angle of $\langle 100\rangle$ axis is less than $0.17 \mathrm{rad}$ ) were present. Comparing with the inverse pole figure shown in Fig. 2(b), it can be seen that the orientation of these grains are mainly the major textural component of

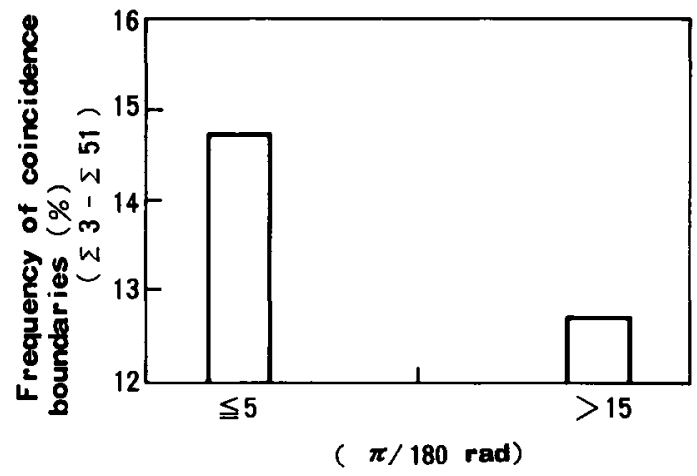

Rotation angle of $\{110\}<001>$ grain about ND axis

Fig. 7. Frequency of coincidence boundaries ( $\Sigma 3-\Sigma 51)$ directly contacted to nearly $\{110\}\langle 001\rangle$ oriented grains and $\{110\}$ grains rotated larger than $0.28 \mathrm{rad}$ around the ND axis present $30 \mu \mathrm{m}$ below the surface of the primary recrystallized specimen (specimen $\mathrm{A}$ ).

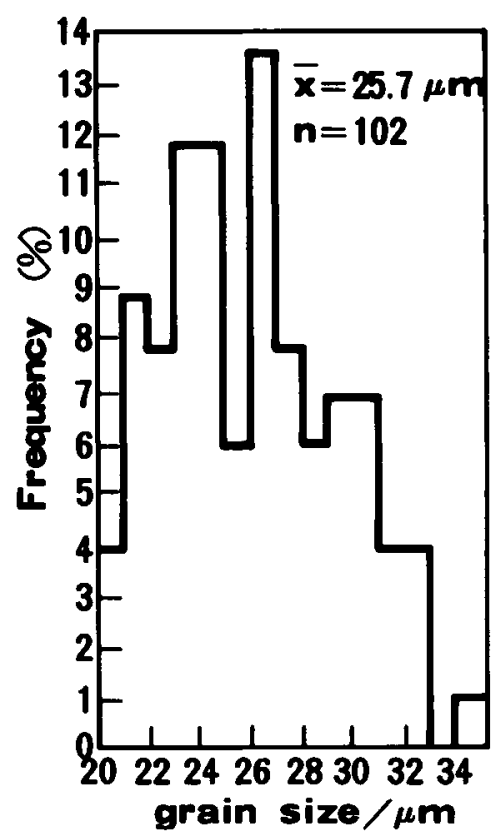

Fig. 8. Orientation and size distribution of matrix grains larger than $20 \mu \mathrm{m}$ in diameter present 30 $\mu \mathrm{m}$ below the surface of the primary recrystallized specimen (specimen $\mathrm{A}$ ). 

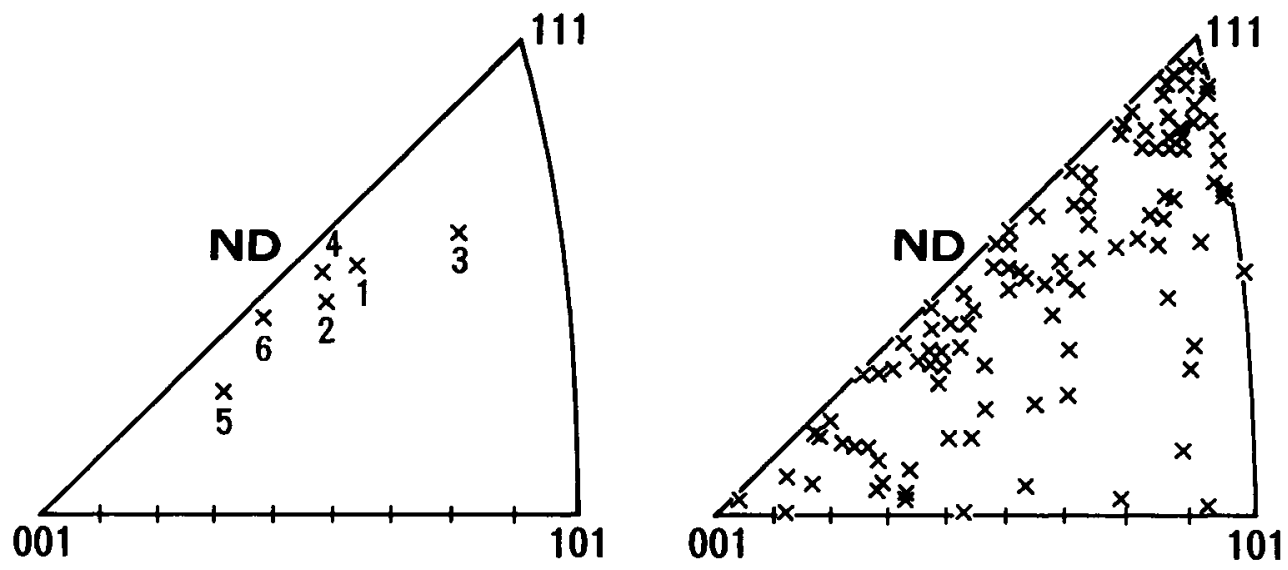

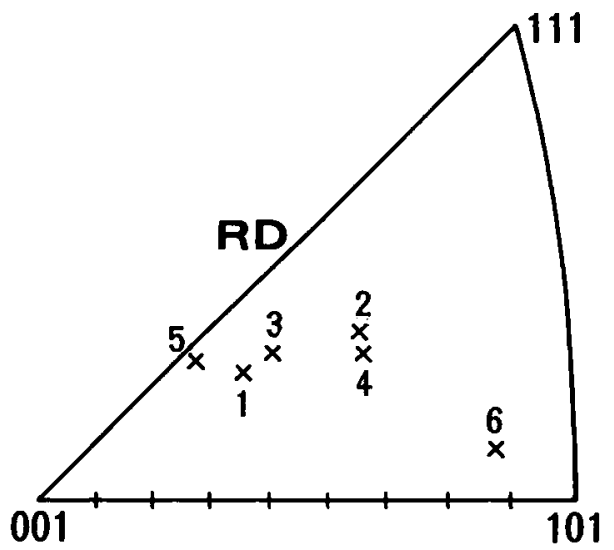

(a) large grains

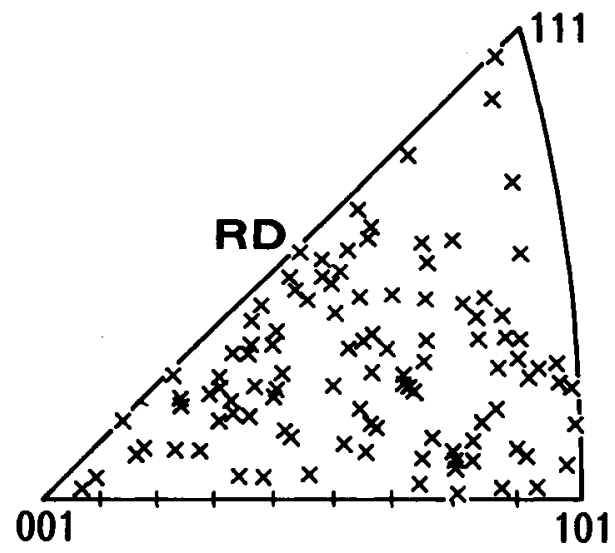

(b) surrounding grains

Fig. 9. An example showing (a) orientations of 6 large grains arbitrarily chosen and (b) 116 grains directly contacted to these grains present at the $30 \mu \mathrm{m}$ below the surface of the primary recrystallized specimen (specimen $\mathrm{A}$ ).

specimen $B$.

Among large grains detected, 6 especially large grains were arbitrarily chosen and orientation of the surrounding grains of these large grains and coincidence orientation relationship of these grains with these large grains were investigated. The number of the surrounding grains were 116 indicating that these large grains are surrounded by 19.3 grains in average. Figure 9 shows orientations of these large grains and surrounding grains. It can be seen that the orientation of these surrounding grains are similar as those surrounding Goss grains shown in Fig. 5.

Figure 10 shows the frequency of coincidence boundaries surrounding large grains.
It can be seen from this figure that the frequency of $\Sigma 9, \Sigma 19$ b $\Sigma 25$ b and $\Sigma 29$ b are relatively high. While the frequency of $\Sigma 1$ boundary of the surrounding grains of $\{110\}$ orientation was low $(2.5 \%)$, the frequency of $\Sigma 1$ boundaries surrounding large grains are high $(6 \%)$ as shown in Fig. 1. Figure 11 shows an example of a microstructure of a large grain and its surrounding grains and their orientations. The size of this large grain is approximately $40 \mu \mathrm{m}$ ( 5 times larger than the average grain size) and it can be a nucleus of secondary recrystallization if the size effect alone is effective for the secondary recrystallization. 


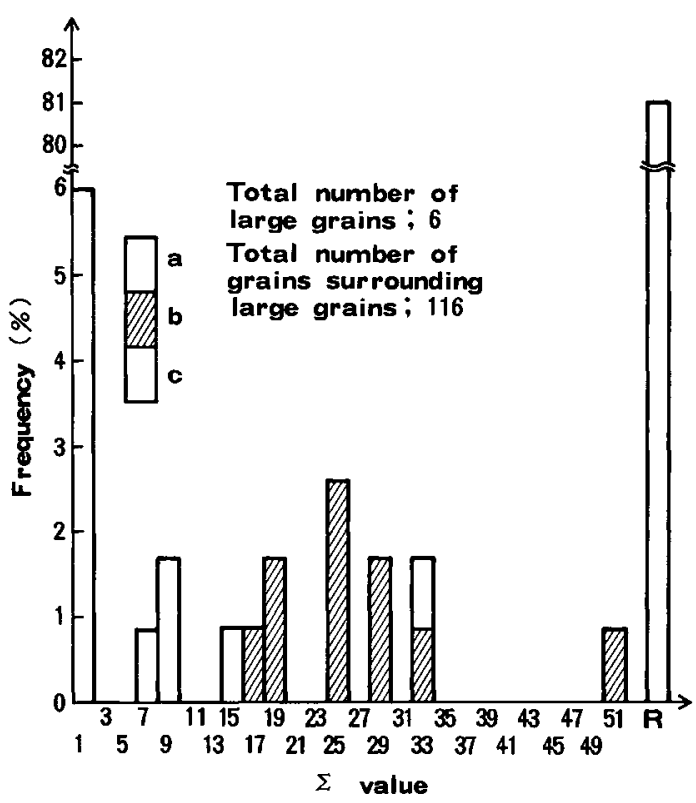

Fig. 10. Frequency of coincidence boundaries surrounding large grains present $30 \mu \mathrm{m}$ below the surface of the primary recrystallized specimen (specimen $\mathrm{A}$ ).

\section{Discussion}

Many investigations have been carried out so far on the distribution of Goss grains in the primary recrystallized specimens ${ }^{(2)(6)-(8)(11)}$. Especially on the two-stage cold rolling method $^{(16)}$, detailed studies were done by Inokuti et $a l .^{(6)}$ utilizing the Kossel method. On the intensity distribution of Goss grains in the primary recrystallized specimen processed by one stage cold rolling process, Flowers and Heckler ${ }^{(11)}$ reported that the intensity of $\{110\}\langle 001\rangle$ oriented grains decreases with increasing deviation angle of $\langle 001\rangle$ axis from the rolling direction through the investigation by the harmonic method ${ }^{(17)}$. This is the same tendency with the present investigation by ECP or the vector method. Takashima et $a ._{.}^{(2)}$ investigated by micro-etch pit method the existence of $\{110\}\langle 001\rangle$ oriented grains in the surface layer of primary recrystallized specimen processed by the one-stage cold rolling process and found that $\{110\}\langle 001\rangle$ oriented grains are often found as colonies.

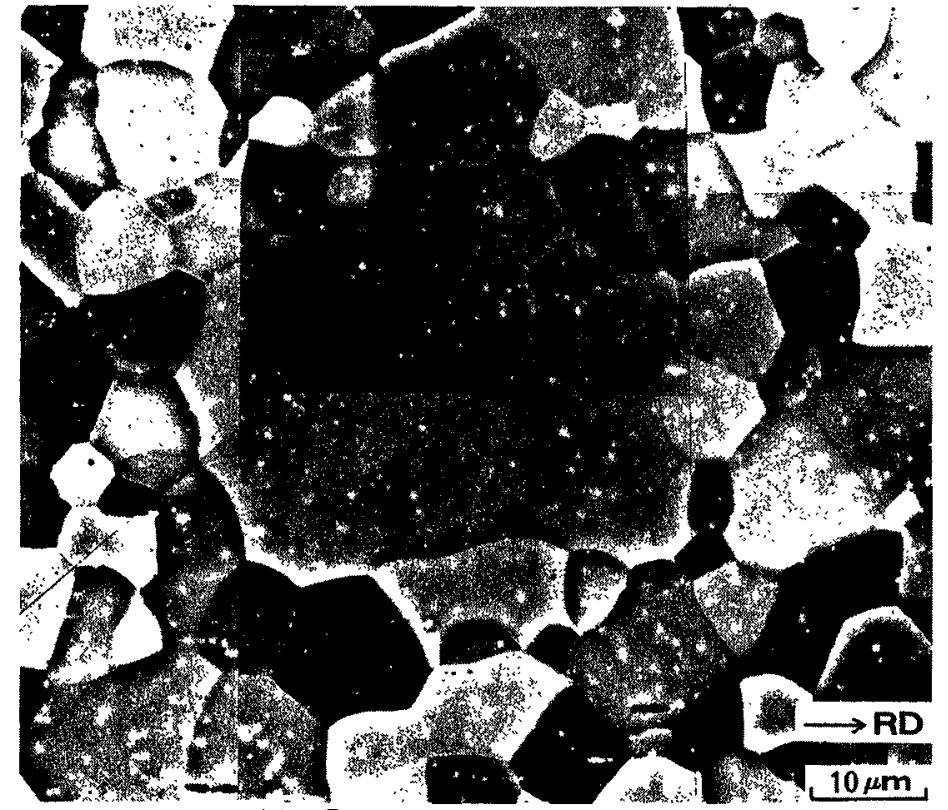

(a) Structure

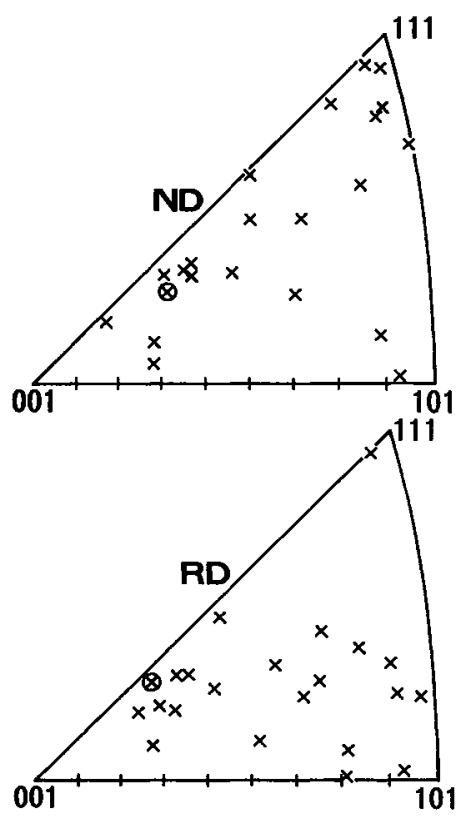

(b) Orientations

Fig. 11. An example showing (a) a microstructure of a large grain and (b) its surrounding grains and their orientations present $30 \mu \mathrm{m}$ below the surface of the primary recrystallized specimen (specimen $\mathrm{A}$ ), $\otimes$ indicates a large grain and $\times$ indicates surrounding grains. 
Sakai et al. ${ }^{(7)}$ reported that $\{110\}\langle 001\rangle$ oriented grains are the largest grains present in the surface layer of the primary recrystallized specimen processed by the one-stage cold rolling method. The present investigation is different from the results obtained by Takashima et al. ${ }^{(2)}$ or Sakai et al. ${ }^{(7)}$. It is not clear why the different results were obtained in the present investigation. One of the reasons may be attributed to the difference of the observed area. The actual amount of the grains having the Goss orientation present in the surface of the primary recrystallized specimen is very small and that the area observed in this experiment is restricted. The grain size of the secondary recrystallization is between 20 and 30 $\mathrm{mm}$ in diameter. It means that the probability of the presence of the potential nucleus for the Goss secondary is null or only one in the area investigated in the present experiment if a secondary grain originates from the one nucleus having the largest grain size in that area in the primary recrystallized specimen. Therefore there is a probability that the potential Goss nucleus was not present in the area investigated by the present experiment.

The following two additional experiments were carried out to investigate that the potential nucleus for secondary recrystallization should always be the largest grains at the primary recrystallized stage. If the potential nuclei of the Goss secondaries are the largest grains, the sharpness of secondaries might be decreased when the specimen size is smaller than the grain size of the secondary recrystallized grain that might grow when the specimen size is large enough.

Figure $12^{(18)}$ shows the effect of the specimen width on the sharpness of Goss secondaries utilizing the similar specimen with the present experiment. The details of the secondary recrytallization annealing condition is shown elsewhere ${ }^{(19)}$. It shows that the sharpness of Goss secondaries does not depend on the specimen width. It was found out in some of the secondary grains that orientations are slightly different from place to place within one grain $^{(20)}$. This suggests that a nucleus of a secondary recrystallized grain might not always originate from only one nucleus grain. These two additional experiments suggest that the potential nucleus of Goss secondaries is not necessarily the largest grains in the primary recrystallized matrix.

Recent finding by Makita et al, ${ }^{(21)}$ in FCC alloys also show that the grains having an orientation identical to the secondary recrystallized grains in the primary matrix are nearly the same in grain size as that of the surrounding grains. The reason that the large grains at the completion of the primary recrystallization cannot be a viable nucleus of secondary

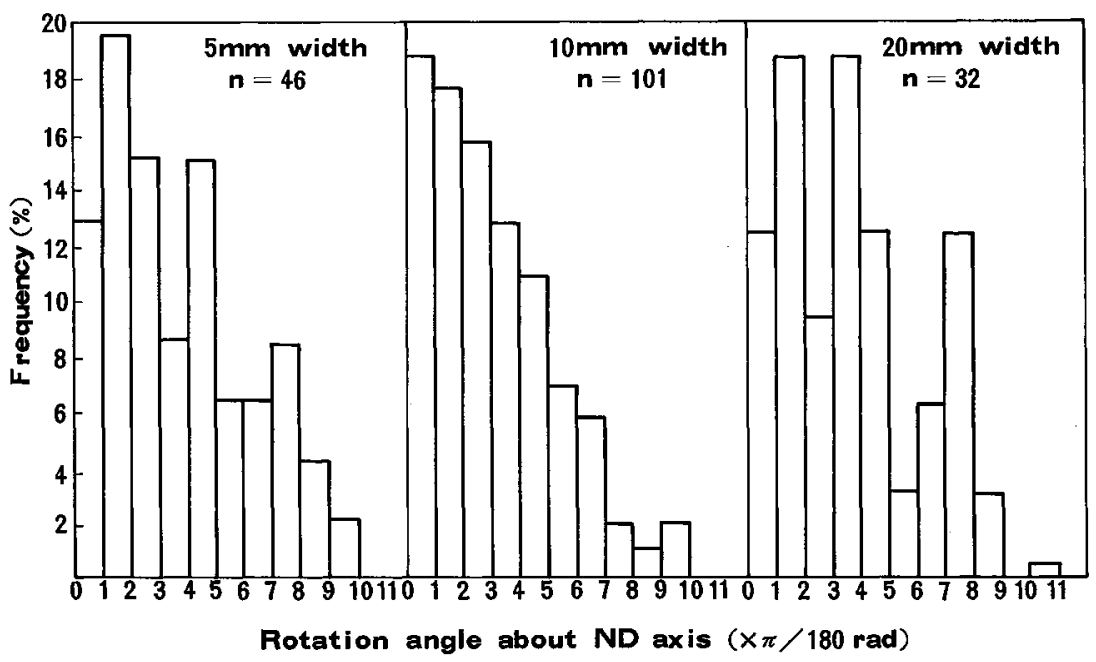

Fig. 12. Effect of the width of the primary recrystallized specimen on the sharpness of Goss secondaries. 
recrystallization might be considered as follows. The present authors indicated that the $\Sigma 1$ boundary is not mobile in the grain growth process of $3 \% \mathrm{Si}-\mathrm{Fe}^{(22)}$. It has also been shown that the $\Sigma 1$ boundary is not mobile and works as an inhibitor for grain growth in FCC alloys $^{(23)}$.

The present investigation shows that large grains present at the primary recrystallized stage has a higher frequency of directly contacing with the $\Sigma 1$ boundary compared with Goss oriented grains. In order to know the probability of these grains to contact with the $\Sigma 1$ boundary in the course of grain growth of these grains, the frequency of the coincidence boundaries corresponding to these 6 large grains in the primary recrystallized matrix were investigated. The orientations of the 507 grains in the primary recrystallized specimen processed by a similar condition to that in the present experiment were utilized as matrix grains for this investigation. (111)[112], (001)[010] and an ideal Goss orientation are also investigated for comparison. (111)[112], (001) [010] are one of the major texture components of the primary texture and an ideal Goss orientation is the major secondary textural component. It can be seen from Fig. 13 that these 6 grains have a higher frequency of the $\Sigma 1$ boundary compared with an ideal Goss orientation, and also that (111)[112], (001)[010] have a higher frequency of the $\Sigma 1$ boundary compared with an ideal Goss orientation.

These results indirectly suggest that grains having these orientations have a higher probability of grain growth inhibition during grain growth in the course of the secondary recrystallization annealing than the Goss orientation. Recently several researchers ${ }^{(22)(24)-(26)}$ have reported that the coincidence boundaries play an important role in the secondary recrystallization of $3 \% \mathrm{Si}-\mathrm{Fe}$ as is the case with FCC alloys ${ }^{(21)(22)(27)}$. It has been shown that an ideal Goss orientation has the highest probability of contacting with the coincidence oriented grains in the course of grain growth ${ }^{(24)}$, and also that coincidence boundaris are more mobile than random boundaries in the grain growth process of the same material ${ }^{(22)}$. It has also been shown that the secondary recrystal-
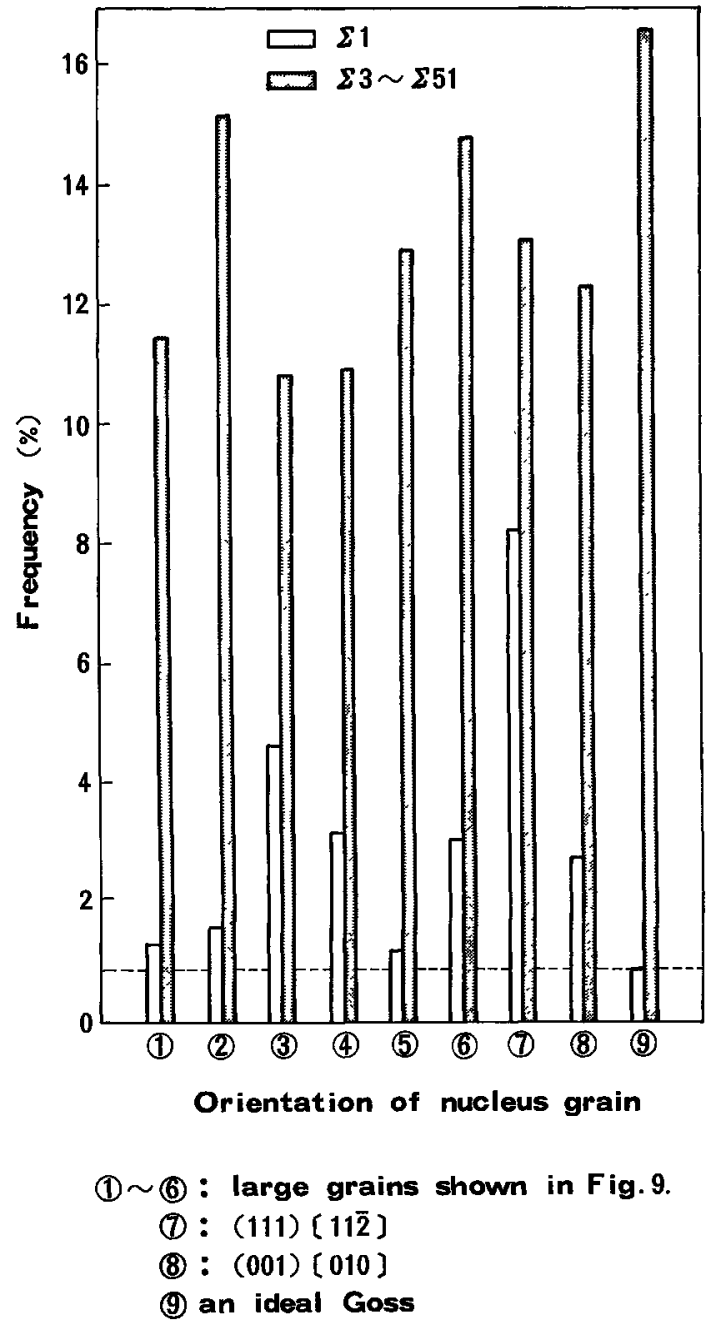

The number of matrix grains are 507.

Fig. 13. Frequency of the $\Sigma 1$ boundary and the coincidence boundaries in the primary recrystallized matrix corresponding to 6 extraordinary large grains and an ideal Goss orientation and the two major orientations ((111)[112], (001)[010]).

lization occurs at the specific ranges of $\mathrm{AlN}$ precipitation $^{(10)}$. It can be considered on the basis of these findings that while the grain boundary migration of the major textural component or larger grains is inhibited by the $\Sigma 1$ boundary or less frequent chances of having coincidence boundaries (except $\Sigma 1$ boundary) around them, some of the Goss oriented grains grow consuming selectively the coincidence oriented grains around them at the specific in- 
tensity level of the inhibitor at which the coincidence boundary could migrate in preference to other boundaries, resulting in the formation of viable nucleus of secondary recrystallization. Based on this mechanism, the size of the nucleus is not the prime necessity for the evolution of the secondary recrystallization texture.

\section{Conclusion}

(1) $\{110\}\langle 001\rangle$ oriented grains present below $30 \mu \mathrm{m}$ from the surface of the primary recrystallized specimen processed by the onestage cold rolling method were not among the largest grains nor were present as an aggregate.

(2) The orientation of the extraordinarily large grains present below $30 \mu \mathrm{m}$ from the surface of the primary recrystallized specimen processed by the one-stage cold rolling method was on the whole similar to that of the major textural component of the primary recrystallized specimen.

(3) Among the $\{110\}$ oriented grains, the Goss oriented grain had the highest frequency of coincidence oriented grains in the grains directly surrounding to these $\{110\}$ oriented grains and had a very low frequency of the $\Sigma 1$ boundary in relation to its orientation in the surface of the primary recrystallized matrix, while the large grains above mentioned had rather higher frequency of the $\Sigma 1$ boundary in relation to its orientation.

(4) The mechanism of the sharp Goss texture formation by secondary recrystallization can be explained successfully, even if the Goss nucleus is not the largest at the completion of the primary recrystallization, by considering the special distribution of coincidence boundaries and their specific gain boundary migration characteristics associated with the intensity of the inhibitor.

\section{REFERENCES}

(1) J. E. May and D. Turnbull: Trans. Met. Soc. AIME, 212 (1958), 769.

(2) K. Takashima, T. Sato and F. Matsumoto: AIP
Conf. Proc., 29 (1975), 566.

(3) H. E. Grenoble: IEEE Trans. on Mag. Mag.-13 (1977), 1425.

(4) M. Shinozaki, I. Matoba, T. Kan and T. Gotoh: Trans. JIM, 19 (1978), 85.

(5) T. Sakai, M. Shiozaki and K. Takashina: J. Appl. Phys. 50(3), (1979), 2369.

(6) Y. Inokuti, G. Maeda, Y. Ito and H. Shimanaka: The Sixth Intern. Conf. on Textures of Materials, II (1981), p. 948.

(7) T. Sakai, M. Matuo, M. Tanino, T. Shindo and M. Shiozaki: The Sixth Intern. Conf. on Textures of Materials, II (1981), p. 938.

(8) N. C. Pease, D. W. Jones, M. H. L. Wise and W. B. Hutchinson: Met. Sci., 15 (1981), 203.

(9) S. Mishira, C. Därmann and K. Lücke: Acta Metall., 32 (1984), 2185.

(10) J. Harase, R. Shimizu K. Takashima and T. Watanabe: Trans. ISIJ, 27 (1987), 965.

(11) J. W. Flowers and A. J. Heckler: IEEE Trans. Magn., M12 (1976), 846.

(12) S. Taguchi, A. Sakakura and H. Takashima: U.S. Patent 3287183 (1966).

(13) D. G. Brandon: Acta Metall., 14 (1966), 1479.

(14) D. Ruer, A. Vadon and B. Baro: Texture of Crystallite Solids, 3 (1979), 245.

(15) R. Shimizu, J. Harase and K. Ohta: Tetsu-toHagané, 73 (1987) S523.

(16) N. P. Goss: Trans. Amer Soc. Metals, 23 (1935), 511.

(17) R. J. Roe: J. Appl. Phys., 36 (1965), 2024.

(18) T. Nozawa: unpublished.

(19) T. Nozawa, T. Nakayama, U. Ushigami and T. Yamamoto: J. of Mag. and Mag. Mat., 58 (1986), 67.

(20) J. Harase and R. Shimizu: unpublished.

(21) H. Makita, S. Hanada, O. Izumi and H. Hukuda: J. Japan Inst. Metals, 51 (1987), 883.

(22) J. Harase, R. Shimizu and T. Watanabe: Proc. of the 7th Riso Intern. Symp. on Metallurgy and Materials Science, (1986), p. 343.

(23) J. Harase, R. Shimizu and T. Watanabe: to be published in the Proc. of the 8th Intern. Conf. on Textures of Materials.

(24) J. Harase, R. Shimizu, K. Kuroki, T. Nakayama and T. Watanabe: Proc. of Grain Boundary Structure and Related Phenomena, Supplement to Trans. JIM., 27 (1986), p. 563.

(25) N. Rouge, G. Vinga and R. Penelle: Proc. of the 7 th Riso Intern. Symp. on Metallurgy and Materials Science, (1986), p. 521.

(26) T. Nakayama and Y. Ushigami: Proc. of the 7th Riso Intern. Symp. on Metallurgy and Materials Science, (1986), p. 463.

(27) M. L. Kronberg and F. H. Wilson: Trans. AIME, 185 (1949), 501. 